\title{
Efecto de la dieta de cerdos en crecimiento sobre el valor nutritivo y la aptitud tecnológica de la carne y grasa
}

\author{
Capra, G. ${ }^{(1) *}$, Repiso, L. ${ }^{(2)}$, Fradiletti, F. ${ }^{(1)}$, Martínez, R. ${ }^{(3)}$, Cozzano, S. ${ }^{(4)}$, Márquez, R. ${ }^{(2)}$ \\ (1) Instituto Nacional de Investigación Agropecuaria, INIA Las Brujas - (2) Gerencia de Proyectos Alimentarios. Labo- \\ ratorio Tecnológico del Uruguay (12/12/1997-31/01/2011), LATU - ${ }^{(3)}$ Facultad de Enfermería y Tecnologías de la Salud, \\ Universidad Católica del Uruguay - ${ }^{(4)}$ Facultad de Ingeniería y Tecnologías, Universidad Católica del Uruguay.
}

Contacto: gcapra@lb.inia.org.uy

Recibido: 30/06/2011 - Aprobado: 1/11/2011

\begin{abstract}
Resumen
Se sometieron cerdos con peso medio inicial de $53 \mathrm{~kg}$ a tres tratamientos: T1) dieta testigo conteniendo suplementos proteicos de origen vegetal y animal, $\mathrm{T} 2$ ) dieta basada exclusivamente en suplementos proteicos de origen vegetal y $\mathrm{T3}$ ) dieta testigo restringida al $90 \%$ de la cantidad ofrecida al T1 más libre acceso a pasturas. Se determinaron parámetros de comportamiento productivo y características de la canal en planta de faena. Se determinaron contenido de grasa intramuscular del Longissimus dorsi, perfil lipídico de la grasa subcutánea e intramuscular, contenido de hierro, zinc, magnesio, sodio y vitamina E. Se determinó también el punto de fusión de la grasa dorsal como indicador de aptitud tecnológica para la elaboración de productos fermentados. Con grasa procedente de cada tratamiento se elaboraron salames que fueron evaluados sensorialmente. En los parámetros de calidad de canal evaluados no hubo diferencias estadísticamente significativas. El acceso a pasturas determinó un incremento en el contenido de ácido linolénico y una mejora de la relación n6/n3 en la grasa subcutánea. No hubo diferencias significativas en el punto de fusión de la grasa. Las muestras de salame resultaron ser distintas, con un nivel de confianza del $95 \%$ para los atributos textura e intención de compra.
\end{abstract}

Palabras clave: Cerdos, composición dieta, calidad carne, aptitud tecnológica, salames.

\begin{abstract}
Pigs with an initial average weight of $53 \mathrm{~kg}$ were allocated to three feeding treatments: T1) control diet containing vegetal and animal protein supplements, T2) diet of only vegetal protein supplements and T3) control diet restricted to $90 \%$ of the amount offered to T1 plus free access to pasture. Productive performance parameters and carcass traits at slaughter were determined. Intramuscular fat content of Longissimus dorsi, lipid profile of the subcutaneous and intramuscular fat, content of iron, zinc, magnesium, sodium and vitamin $E$ were analyzed. The melting point of the back fat was also analyzed as an indicator of the technological aptitude for the manufacturing of fermented products. Fat from each treatment was used to manufacture salamis that were sensory evaluated. Carcass quality parameters did not show statistically significant differences. Access to pasture showed an increase in linolenic acid content and a better relationship $n 6 / n 3$ in the subcutaneous fat. There were no significant differences in the fat melting point. Salami samples were different with a confidence level of $95 \%$ for texture attributes and purchase intent. Keywords: Pigs, diet composition, meat quality, technological aptitude, salami.
\end{abstract}

\section{Introducción}

Este estudio se realizó en el marco del Programa Nacional de Producción Familiar del INIA, en un proyecto de investigación que tiene como objetivo general valorizar productos cárnicos de la producción familiar por medio del desarrollo de tecnología de producción y procesos de transformación que contribuyan a su diferenciación.

Según estimaciones realizadas en base al Censo Agropecuario del año 2000, la producción familiar constituye el $79 \%$ de las explotaciones de Uruguay. En el subsector porcino el $84 \%$ de los establecimientos que tienen a este rubro como principal fuente de ingresos corresponde a pequeños productores familiares, aunque sólo contribuyen con un $22 \%$ del Valor Bruto de Producción de ese rubro productivo (IICA, 2007). En las dos últimas décadas se ha verificado una drástica reducción del número de establecimientos dedicados a la producción de cerdos, a la vez que se produce un proceso de concentración en unidades productivas de mayor tamaño.
Cifras oficiales señalan que en el período comprendido entre los años 2000 y 2006 se produjo una reducción del $54 \%$ en el número de explotaciones dedicadas a la producción comercial de cerdos; el cese de la actividad se produjo principalmente en las unidades productivas de menor tamaño, ya que un $90 \%$ de las que abandonaron el rubro eran explotaciones con menos de 50 porcinos (DIEA-MGAP e INIA, 2007). Uno de los factores determinantes de la reducción en el número de establecimientos dedicados al rubro y en el total de animales faenados ha sido la competencia del producto importado. En la actualidad se importa casi la mitad del volumen de carne y grasa requeridos por la industria chacinera (Errea, 2010).

La demanda en el mercado interno de cortes frescos de carne porcina presenta un crecimiento sostenido. De acuerdo a información estadística del Instituto Nacional de Carnes, el volumen comercializado de carne porcina destinada al abasto de la ciudad de Montevideo se incrementó un $23 \%$ entre 2009 y 2010 (INAC, 2011). Esta evolución determina la necesidad de profundizar conocimientos sobre el aporte nutritivo de la carne obtenida en condiciones prevalecientes a nivel de 
la producción familiar, con el fin de promover el consumo y propender a una mejor inserción de los pequeños productores en la cadena comercial.

Paralelamente, la industria de los chacinados muestra preocupación por la baja calidad de la grasa de origen nacional, para lo cual se entiende necesario realizar una evaluación del comportamiento de la materia prima obtenida en esas condiciones de producción. Una importante proporción de los industriales chacineros ha identificado como principal problema tecnológico, vinculado a la materia prima de origen nacional, la calidad de la grasa (Echenique y Capra, 2007). La problemática se relaciona al comportamiento térmico de la grasa, determinado por su composición química, que provoca efectos negativos sobre las cualidades de embutidos fermentados (Grompone e Irigaray, 2008). Echenique (2007) sostiene que desde el punto de vista tecnológico altos niveles de ácidos grasos poliinsaturados generan problemas en la elaboración de productos fermentados crudos, dada la consistencia blanda de la grasa, la fluidización durante la fase de fermentación y los problemas de enranciamiento. En condiciones reales de producción de nuestro país se ha constatado que los niveles totales de ácidos grasos poliinsaturados de la grasa porcina se ubican en un rango muy amplio, comprendido entre 10,8 a 28,3\% (Echenique y Capra, 2006). En condiciones experimentales, mediante cambios en la composición de la dieta de cerdos en engorde, se han provocado variaciones en el contenido de ácido linoleico comprendidas entre el 12,9 y el $18,0 \%$ (Capra et al., 2007).

El objetivo específico de este trabajo fue evaluar el efecto del sistema de producción y la composición de la dieta sobre las cualidades nutricionales de la carne y la aptitud tecnológica de la grasa para la elaboración de productos fermentados. Para esto se diseñaron dietas para cerdos en crecimiento-terminación representativas de aquellas a las que normalmente acceden los productores familiares uruguayos. Se compararon tres tratamientos alimenticios, evaluándose parámetros de comportamiento productivo, características de la canal en planta de faena y atributos de la carne y grasa en laboratorio. Se tomaron muestras de carne y grasa para la determinación del contenido de grasa intramuscular del músculo Longissimus dorsi, perfil lipídico de la grasa subcutánea e intramuscular, contenido de hierro, zinc, magnesio, sodio y vitamina E. También se determinó el punto de fusión de la grasa subcutánea, como indicador de la aptitud tecnológica para la elaboración de productos fermentados. Con la grasa obtenida en cada uno de los tratamientos se fabricaron salames en una industria chacinera, a los cuales se les realizaron análisis fisico-químicos y sensoriales.

\section{Materiales y Métodos}

\section{Animales}

Se utilizaron 36 cerdos cuyo biotipo corresponde a cruzamientos recurrentes entre Landrace y Large White, que al inicio del estudio pesaban en promedio 53,4 kg. Los animales fueron asignados al azar a los tratamientos, con igual número de machos y hembras en cada uno de ellos.

\section{Alimentación de los animales}

Se compararon tres tratamientos de alimentación consistentes en:

T1- dieta testigo conteniendo suplementos proteicos de origen vegetal y animal (ración balanceada R1).

T2- dieta basada exclusivamente en suplementos proteicos de origen vegetal (ración balanceada R2).

T3- dieta testigo restringida al $90 \%$ del T1 (con ración balanceada R1) más acceso libre a pasturas.

Las raciones balanceadas R1 y R2 fueron formuladas en base a tablas de requerimientos nutricionales de los cerdos en crecimiento (NRC, 1998) y tablas de composición de ingredientes de dietas para cerdos (Fedna, 2003), de modo tal que cumplieran con la condición de ser isocalóricas (3,2 Mcal $/ \mathrm{kg}$ ) e isoproteicas (15\% proteína cruda en base fresca). Se ajustaron a la formulación indicada en la Tabla 1, que resultó en la composición química detallada en la Tabla 2.

\begin{tabular}{|l|c|c|}
\hline Ingrediente (en \%) & R1 & R2 \\
\hline Maíz & 79,8 & 64,8 \\
\hline Afrechillo de arroz desgrasado & -- & 15,0 \\
\hline Harina de soja & 12,0 & 15,0 \\
\hline Harina de carne & 7,5 & -- \\
\hline Fosfato monosódico de dihidrógeno & -- & 2,0 \\
\hline Carbonato de calcio & -- & 2,5 \\
\hline L Lisina & 0,1 & 0,1 \\
\hline Cloruro de sodio & 0,5 & 0,5 \\
\hline Núcleo vitamínico mineral & 0,1 & 0,1 \\
\hline
\end{tabular}

Tabla 1. Fórmula de las raciones utilizadas.

\begin{tabular}{|l|c|c|}
\hline & R1 & R2 \\
\hline Materia seca & 86,6 & 86,2 \\
\hline Proteína cruda* & 16,1 & 16,9 \\
\hline Fibra cruda* & 2,0 & 2,0 \\
\hline Grasas y aceites* & 5,4 & 3,9 \\
\hline Cenizas* & 5,9 & 6,9 \\
\hline Calcio* & 1,1 & 1,0 \\
\hline Fósforo total* & 0,9 & 1,0 \\
\hline
\end{tabular}

Tabla 2. Composición química de las raciones en porcentaje (* Datos expresados en base seca).

La composición química de estas raciones fue determinada previo a este estudio, en base a los siguientes métodos analíticos: Materia seca según AOAC 930.15, proteínas según AOAC 988.05, fibra cruda según AOAC 962.09, aceites y grasas según AOAC 920.39, cenizas según AOAC 942.09, calcio según AOAC 927.02, fósforo según AOAC 969.31

El suministro diario de alimento balanceado se ajustó a una escala basada en el peso vivo, de acuerdo al detalle expresado en la Tabla 3.

\begin{tabular}{|c|c|c|}
\hline Peso vivo (kg) & Tratamientos T1 y T3 & Tratamientos T2 \\
\hline $\mathbf{3 0}-\mathbf{4 0}$ & 1,55 & 1,40 \\
\hline $\mathbf{4 0}-\mathbf{5 0}$ & 1,90 & 1,70 \\
\hline $\mathbf{5 0}-\mathbf{6 0}$ & 2,20 & 2,00 \\
\hline $\mathbf{6 0}-\mathbf{7 0}$ & 2,50 & 2,25 \\
\hline $\mathbf{7 0}-\mathbf{8 0}$ & 2,75 & 2,50 \\
\hline $\mathbf{8 0}-\mathbf{9 0}$ & 2,90 & 2,60 \\
\hline $\mathbf{9 0}-\mathbf{1 0 0}$ & 3,00 & 2,70 \\
\hline $\mathbf{1 0 0}-\mathbf{1 2 0}$ & 3,10 & 2,80 \\
\hline
\end{tabular}

Tabla 3. Asignación diaria de alimento balanceado en kilogramos por animal y por día según peso vivo (adaptado de Henry et al., 1984). 
El acceso a pastura del T3 fue libre, pero se limitaron con hilo eléctrico franjas semanales de superficie variable, según la evolución del consumo y el estado de la pastura, una pradera de segundo año compuesta por trébol rojo y achicoria. No se determinó el consumo de pastura.

\section{Comportamiento productivo}

Los parámetros de comportamiento productivo que se determinaron fueron la evolución del peso vivo individual y el consumo de alimentos e índice de conversión del lote.

Las características de la canal determinadas en planta de faena fueron:

- $\quad$ el peso de la canal: peso en caliente en segunda balanza de la canal con cabeza, sin grasa peri-renal y sin riñones,

- $\quad$ el largo de la canal: medido con cinta métrica sobre la media res izquierda, desde el borde anterior de la sínfisis isquiopubiana hasta la porción media del borde anterior de la primera costilla,

- $\quad$ el espesor de la grasa dorsal (EGD): promedio del espesor obtenido en dos puntos sobre la línea media medidos con calibre digital, a nivel del m. Gluteus medio (EGD gm) y de la última costilla (EGD uc),

- el espesor del músculo lumbar en el punto $\mathrm{M}$ medido con calibre digital (ancho M): distancia mínima entre el borde craneal del músculo Gluteus medius y el eje dorsal del canal vertebral (Daumas, 2001).

Tanto el espesor de la grasa dorsal como el del músculo lumbar en el punto $\mathrm{M}$ son utilizados para tipificación de canales porcinas en mataderos pequeños de algunos países de Europa (Método ZP o ZweiPunkte).

Las muestras de carne para análisis de grasa intramuscular fueron tomadas del músculo Longissimus dorsi, extraídas a nivel de la $3^{\mathrm{a}}$ y $4^{\mathrm{a}}$ últimas costillas.

Las muestras de grasa subcutánea se tomaron en dos puntos de la línea media de la canal, ubicados a la misma altura donde se realiza la determinación del espesor de la grasa dorsal (Figura 1).

\section{Elaboración de los salames}

Se elaboraron salames tipo Milán con el objetivo de utilizar la grasa de los animales de este estudio en un producto que pudiera incluirla sin ningún tratamiento previo. Para la elaboración de los salames se utilizó la fórmula de la industria que colaboró en esta investigación, que contiene $30 \%$ de grasa firme de cerdo. Se elaboraron tres lotes de $100 \mathrm{~kg}$ cada uno, con grasa de cada tratamiento y utilizando carne de toro. Se embutieron en fundas de colágeno de $120 \mathrm{~mm}$ de diámetro y fueron colocados en estufa a $20^{\circ} \mathrm{C}$ y 80 a $90 \%$ de humedad relativa durante 2 días. Al tercer día se colocaron en estufa a $24^{\circ} \mathrm{C}$ y 80 a $90 \%$ de humedad relativa. Al cuarto día se pasaron a secaderos con $13{ }^{\circ} \mathrm{C}$ de temperatura y $75 \%$ de humedad relativa, donde permanecieron hasta el día 12. Finalmente se analizó $\mathrm{pH}$, actividad de agua y proteínas en una muestra de cada lote, según la metodología que se indica más adelante.

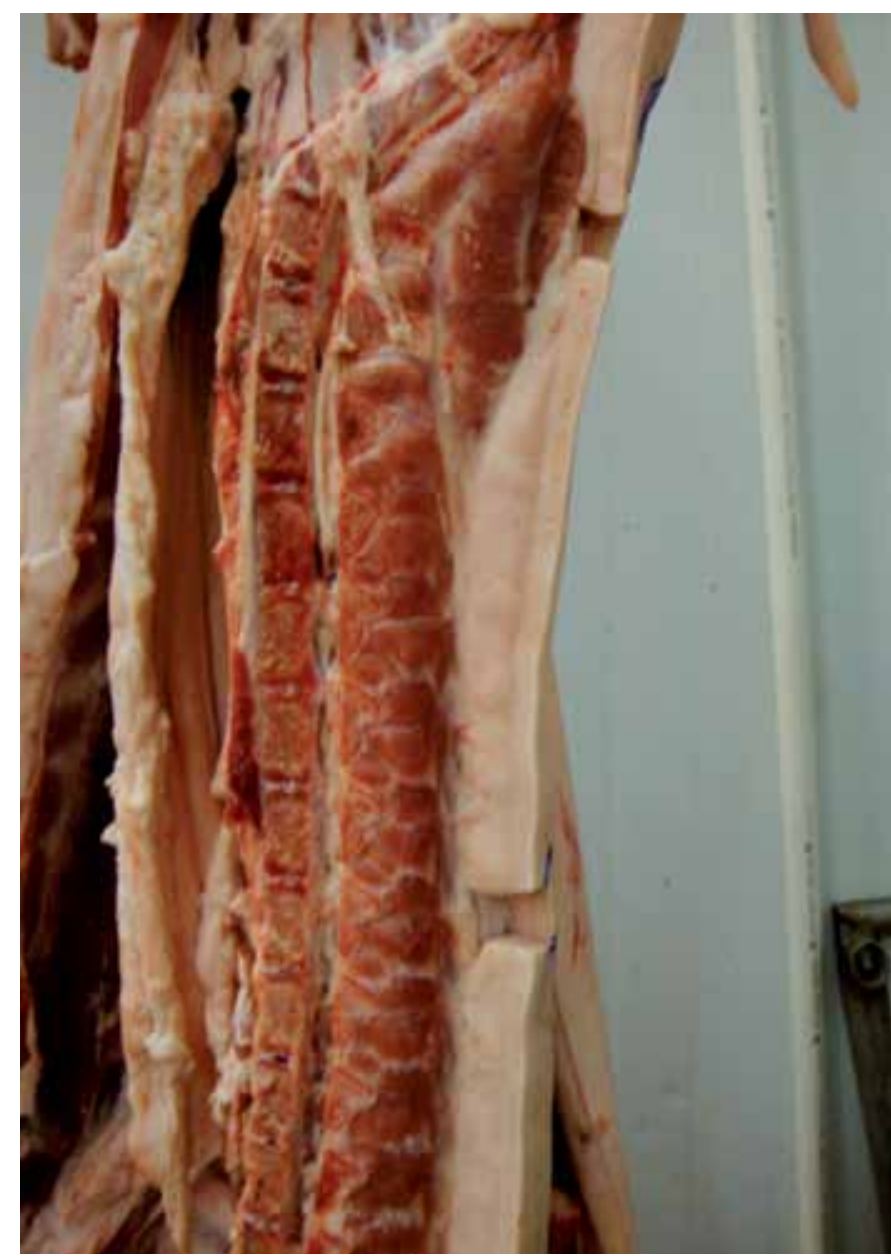

Figura 1. Puntos de la toma de muestra de grasa subcutánea y de medida del EGD.

\section{Determinaciones analíticas}

\section{Perfil lipídico}

Se utilizaron 30 muestras de carne y 30 muestras de grasa subcutánea ( 10 de cada tipo de alimentación), de 30 gramos cada una.

Las muestras fueron trituradas y homogeneizadas en una procesadora doméstica previo a su envasado a vacío en bolsas termocontraíbles de baja permeabilidad al oxígeno y al vapor de agua, y luego fueron congeladas a $-18{ }^{\circ} \mathrm{C}$ hasta el momento de su análisis. Los ensayos se realizaron por duplicado.

La valoración del porcentaje de grasa intramuscular se realizó según el método de Folch, Lees y Sloane (1957).

Los ácidos grasos se determinaron mediante cromatografía de gases y espectrometría de masa, en conformidad con el método de AOCS Oficial Method Ce 2-66, AOCS Ce 1-62, AOCS Ce 1-91.

\section{Relaciones entre componentes del perfil lipídico}

Se calcularon relaciones entre diferentes fracciones que componen las grasas y que normalmente son utilizadas como indicadores de sus cualidades desde el punto de vista nutricional y de sus posibles efectos sobre la salud del consumidor. Entre estas relaciones se incluyen el cociente entre los ácidos grasos poliinsaturados (AGPI) y los saturados (AGS), así como la relación entre los n-6 y los n-3. También se incluyen los índices de aterogenecidad (AI) y trombogenecidad (TI) propuestos por Ulbricht y Southgate (1991) y el cociente h/H entre la sumatoria de los ácidos grasos hipocolesterolémicos (mono y poliinsaturados) y los hipercolesterolémicos (mirístico y palmítico) planteados por Herranz et al. (2008) para la descripción de salames procedentes de diferentes países. 
Las fórmulas de cálculo aplicadas fueron:

$\mathrm{AI}=[\mathrm{C12:0}+(4 * \mathrm{C} 14: 0)+\mathrm{C} 16: 0] /\left[\left(\sum \mathrm{AGPI}\right)+\left(\sum \mathrm{AGMI}\right)\right]$

TI $=\left[\right.$ C14:0 + C16:0 + C18:0] $/\left[\left(0,5^{*} \sum\right.\right.$ AGMI $)+\left(0,5^{*} \sum \mathbf{n}-6\right)+\left(3^{*} \sum \mathbf{n}-3\right)+(\mathbf{n}-3 / \mathbf{n}-6]$

$\mathbf{h} / \mathbf{H}=\left(\sum\right.$ AGMI $+\sum$ AGPI $) /($ C14:0 + C16:0 $)$

Asimismo, se estimó la relación entre el contenido de ácido esteárico y ácido linoleico, que ha sido propuesta como indicador de la aptitud tecnológica de la grasa (Daza y Bouxadé, 2000).

\section{Minerales}

Para el análisis de los minerales se utilizaron 30 muestras de carne de 5 gramos cada una, 10 de cada tipo de alimentación. Las muestras se trituraron en procesadora doméstica, se homogeneizaron, se envasaron a vacío en bolsas termocontraíbles de baja permeabilidad al oxígeno y al vapor de agua, y se congelaron a $-18{ }^{\circ} \mathrm{C}$ hasta realizar el análisis.

Se determinaron hierro, zinc, magnesio y sodio en muestras digeridas en sistema cerrado a alta presión según método AOAC 999.10 adaptado, por emisión atómica (ICP-OES) basado en ISO 11885:1996 adaptada. Los ensayos se realizaron por duplicado.

\section{Vitamina E}

El análisis de vitamina $\mathrm{E}$ se hizo sobre 30 muestras de 30 gramos de carne cada una, 10 de cada tipo de alimentación. Las muestras se trituraron en procesadora doméstica, se homogeneizaron, se envasaron a vacío en bolsas termocontraíbles de baja permeabilidad al oxígeno y al vapor de agua, se cubrieron con papel de aluminio para evitar la exposición a la luz y se congelaron a $-18^{\circ} \mathrm{C}$ hasta su análisis.

El análisis de vitamina E se basó en la norma BS EN 12822, que consta en una saponificación y posterior extracción con solvente orgánico. La capa orgánica se inyectó directamente en HPLC luego de filtrar por 0,22 micras. La separación cromatográfica se hizo en fase normal (Phenomenex Silica) usando un detector de fluorescencia a 290-330 nm. Los ensayos se realizaron por triplicado; si en algún caso el rango normalizado está fuera de los gráficos de control, se realiza por quintuplicado.

\section{Punto de fusión}

El punto de fusión de determinó sobre 30 muestras de grasa subcutánea de $50 \mathrm{~g}$ cada una, 10 de cada tipo de alimentación. El análisis se realizó en base al método AOCS Cc1-25(93) sobre la materia grasa extraída con hexano en equipo Soxtec con capilar cerrado.

\section{Proteínas}

Se determinó el contenido en proteínas de los salames elaborados con grasa de cada tratamiento. El método utilizado se basó en la norma ISO 937:1978.

\section{Actividad de agua}

Se midió la actividad de agua ( $\mathrm{a}_{\mathrm{w}}$ ) tanto en la pasta del salame (durante su elaboración) como en el producto final. La determinación se realizó con un equipo Aqualab Series $3 \mathrm{TE}$ a $25^{\circ} \mathrm{C}$.

\section{pH}

Se midió el pH de los salames elaborados utilizando un medidor de $\mathrm{pH}$ Mettler Toledo SevenMulti S47.

\section{Evaluación sensorial}

Se evaluó textura, agrado general e intención de compra de los salames elaborados con grasa de los diferentes tratamientos. Para la evaluación sensorial de los atributos de textura y agrado general se utilizó una escala hedónica estructurada de nueve puntos (1- Me disgusta mucho, 5- Me es indiferente, 9- Me gusta mucho). Para la evaluación de la intención de compra se utilizó una escala estructurada de siete puntos (1- Definitivamente no lo compraría, 4-Tal vez sí/ Tal vez no, 7-Definitivamente lo compraría). Finalmente se les pidió a los consumidores que indicaran qué muestra preferían y se calculó el porcentaje de preferencia de cada una.

Para la evaluación se convocó a consumidores esporádicos y habituales de salames.

Fueron reclutados 40 consumidores de 20 a 65 años de edad de los cuales $30 \%$ eran de sexo femenino.

Las muestras fueron presentadas en fetas de 15-20 g contenidas en placas plásticas transparentes codificadas con números aleatorios de tres dígitos siguiendo un orden de presentación balanceado para cada participante. Los consumidores contaban con agua sin gas y galletitas cracker sin sal como borradores. La evaluación se realizó en una sala normalizada según ISO 8589:1988 bajo luz artificial blanca y con temperatura controlada (entre 22 y $24^{\circ} \mathrm{C}$ ).

Para el análisis de los resultados se hizo un análisis de varianza para cada atributo, se calculó la diferencia mínima significativa utilizando el test de Tukey $(\alpha=0.05)$ con Infostat versión 2008.

\section{Análisis estadísticos}

Los datos fueron analizados utilizando el procedimiento GLM del SAS (SAS, 2003) con el tratamiento y el sexo incluidos en el modelo como efectos fijos; en parámetros vinculados a las características de la canal se incluyó como covariable el peso de la canal.

\section{Resultados y Discusión}

\section{Comportamiento productivo y características de la canal}

En este estudio no hubo diferencias significativas entre tratamientos en cuanto a espesor de grasa dorsal y contenido de grasa intramuscular, como se muestra en la Tabla 4, a diferencia de ensayos precedentes en los que se ha constatado reducción significativa del contenido de grasa intramuscular en los cerdos con acceso a pasturas (Bauzá et al., 2003; Echenique et al., 2009). Se verificaron diferencias entre tratamientos en la ganancia media diaria y en el peso final alcanzado.

Enfält et al. (1997) afirman que los cerdos criados a campo muestran ganancias de peso menores y carcasas más magras que los cerdos en estabulación. En este estudio no se presentaron diferencias estadísticamente significativas ni en espesor de grasa dorsal ni en contenido de grasa intramuscular. Las ganancias más bajas de los animales con acceso a pastura podrían atribuirse a que el consumo de forraje no lograba compensar la restricción impuesta en la cantidad de alimento balanceado ofrecido. 


\begin{tabular}{|c|c|c|c|c|}
\hline & T1 & T2 & T3 & $P^{*}$ \\
\hline Peso final (kg) & $113,5 \pm 1,3^{a}$ & $104,9 \pm 1,4^{b}$ & $107,4 \pm 1,4^{b}$ & 0,0083 \\
\hline Aumento peso (kg) & $59,8 \pm 1,1^{\mathrm{a}}$ & $53,1 \pm 1,2^{a, b}$ & $50,3 \pm 1,2^{b}$ & $<0,0001$ \\
\hline GMD (kg/día) & $0,792 \pm 0,012^{\mathrm{a}}$ & $0,671 \pm 0,018^{a, b}$ & $0,651 \pm 0,017^{b}$ & $<0,0001$ \\
\hline Peso canal (kg) & $88,5 \pm 1,5^{\mathrm{a}}$ & $81,3 \pm 1,5^{b}$ & $85,3 \pm 1,5^{a, b}$ & 0,0065 \\
\hline Índice de conversión & 3,63 & 4,20 & 3,96 & \\
\hline Largo (mm) & $839 \pm 7^{\mathrm{a}}$ & $859 \pm 7^{\mathrm{a}}$ & $858 \pm 7^{\mathrm{a}}$ & 0,2046 \\
\hline EGD gm (mm) & $21,5 \pm 1,7^{\mathrm{a}}$ & $19,2 \pm 1,8^{a}$ & $17,8 \pm 1,5^{a}$ & 0,1502 \\
\hline EGD uc (mm) & $24,0 \pm 1,3^{\mathrm{a}}$ & $23,0 \pm 1,4^{\mathrm{a}}$ & $21,2 \pm 1,2^{a}$ & 0,1231 \\
\hline EGD medio (mm) & $22,8 \pm 1,3^{\mathrm{a}}$ & $21,1 \pm 1,4^{a}$ & $19,5 \pm 1,2^{a}$ & 0,0765 \\
\hline Ancho M (mm) & $75,7 \pm 2,3^{a}$ & $75,5 \pm 2,5^{\mathrm{a}}$ & $74,5 \pm 2,1^{\mathrm{a}}$ & 0,7124 \\
\hline Punto fusión grasa $\left({ }^{\circ} \mathrm{C}\right)$ & $41,0 \pm 0,5^{\mathrm{a}}$ & $40,9 \pm 0,4^{\mathrm{a}}$ & $40,8 \pm 0,4^{\mathrm{a}}$ & 0,9252 \\
\hline Grasa intramuscular (g/100g) & $2,57 \pm 0,31^{\mathrm{a}}$ & $2,35 \pm 0,29^{a}$ & $2,05 \pm 0,31^{\mathrm{a}}$ & 0,5018 \\
\hline
\end{tabular}

Tabla 4. Parámetros de comportamiento productivo y características de la canal (*P - Nivel de significancia del efecto del tratamiento en el modelo). Letras iguales dentro de una misma fila indican que no existe diferencia significativa para ese parámetro.

\section{Composición de la grasa intramuscular y aporte de micronutrientes de la carne}

\begin{tabular}{|c|c|c|c|c|}
\hline & T1 & T2 & T3 & $\mathbf{P}^{*}$ \\
\hline C14:0 & $1,02 \pm 0,03^{a}$ & $1,02 \pm 0,03^{a}$ & $1,03 \pm 0,03{ }^{a}$ & 0,9487 \\
\hline C16:0 & $24,20 \pm 0,31^{a}$ & $24,12 \pm 0,29^{a}$ & $24,84 \pm 0,31^{\mathrm{a}}$ & 0,2087 \\
\hline C16:1 & $2,32 \pm 0,18^{\mathrm{a}}$ & $2,31 \pm 0,17^{\mathrm{a}}$ & $2,38 \pm 0,18^{\mathrm{a}}$ & 0,9550 \\
\hline C18:0 & $14,63 \pm 0,48^{\mathrm{a}}$ & $15,36 \pm 0,46^{\mathrm{a}}$ & $14,75 \pm 0,48^{\mathrm{a}}$ & 0,5040 \\
\hline C18:1 & $42,64 \pm 0,91^{a}$ & $41,31 \pm 0,87^{\text {a }}$ & $44,06 \pm 0,91^{\mathrm{a}}$ & 0,5734 \\
\hline C18:2 & $9,96 \pm 0,71^{\mathrm{a}}$ & $9,83 \pm 0,68^{\text {a }}$ & $9,73 \pm 0,71^{\mathrm{a}}$ & 0,9746 \\
\hline C18:2 CLA & $0,11 \pm 0,01^{\mathrm{a}}$ & $0,12 \pm 0,01^{\mathrm{a}}$ & $0,14 \pm 0,01^{\mathrm{a}}$ & 0,3913 \\
\hline C18:3 & $0,27 \pm 0,06^{\mathrm{a}}$ & $0,23 \pm 0,06^{\mathrm{a}}$ & $0,44 \pm 0,06^{b}$ & 0,0482 \\
\hline C20:4 & $1,40 \pm 0,32^{\mathrm{a}}$ & $1,85 \pm 0,25^{\mathrm{a}}$ & $1,37 \pm 0,28^{a}$ & 0,3980 \\
\hline$\sum$ AGS & $40,86 \pm 0,71^{\mathrm{a}}$ & $41,48 \pm 0,68^{\mathrm{a}}$ & $41,56 \pm 0,71^{\mathrm{a}}$ & 0,7502 \\
\hline$\sum$ AGMI & $46,46 \pm 1,03^{a}$ & $45,15 \pm 0,99^{\mathrm{a}}$ & $45,84 \pm 1,03^{\text {a }}$ & 0,6574 \\
\hline$\sum$ AGPI & $12,22 \pm 1,07^{\mathrm{a}}$ & $13,23 \pm 1,02^{\mathrm{a}}$ & $12,33 \pm 1,07^{\mathrm{a}}$ & 0,7532 \\
\hline$\sum n-6$ & $11,06 \pm 0,93^{\mathrm{a}}$ & $11,84 \pm 0,88^{\mathrm{a}}$ & $11,04 \pm 0,93^{\mathrm{a}}$ & 0,7723 \\
\hline$\sum \mathbf{n}-\mathbf{3}$ & $0,92 \pm 0,14^{\mathrm{a}}$ & $0,93 \pm 0,13^{\mathrm{a}}$ & $1,01 \pm 0,14^{\mathrm{a}}$ & 0,8810 \\
\hline
\end{tabular}

Tabla 5. Perfil lipídico de la grasa intramuscular expresado en porcentajes $\left({ }^{*} \mathrm{P}\right.$ - Nivel de significancia del efecto del tratamiento en el modelo). Letras iguales dentro de una misma fila indican que no existe diferencia significativa entre las muestras para ese parámetro.

En el tratamiento T3 se logró un aumento significativo del ácido graso n-3 $\alpha$-linolénico a nivel intramuscular respecto a los tratamientos T1 y T2. A pesar de que la diferencia es significativa y teóricamente favorable para los consumidores, debe ser relativizada por su magnitud y por el hecho de que existen otras fuentes alternativas de aporte de estos ácidos grasos, aunque para nuestro país las mismas son de bajo consumo. El contenido de ácido graso $n-6$ linoleico intramuscular no presentó diferencias significativas entre los tratamientos y tampoco se obtuvieron diferencias en la relación n-6/n-3. Dado que la grasa intramuscular es de consumo inevitable, es relevante desde el punto de vista nutricional valorar el mayor aporte de n-3 $\alpha$-linolénico sin aumentar el contenido de n-6 linoleico en la porción de carne a recomendar, más que evaluar la relación n-6/n-3. Sin embargo, el incremento del n-3 $\alpha$-linolénico constituye uno de los objetivos desde el punto de vista nutricional ya que posibilita la síntesis de eicosanoides que otorgan importantes beneficios para la salud de los consumidores, destacándose su funcionalidad antihemostática, antitrombótica y de mantenimiento de la fluidez de las membranas celulares (Coronado et al., 2006).

Vautier (2005), en una amplia revisión de factores que determinan variaciones en el valor nutritivo de la carne de cerdo, establece que hay resultados contradictorios en cuanto al efecto del sistema de producción sobre el contenido de grasa intramuscular, pero señala el efecto incremental del sistema de producción a campo sobre el contenido de $\Sigma$ AGPI y de ácido $\alpha$-linolénico en comparación con la producción en confinamiento. Considerando que en este estudio el tratamiento T1 es estabulado y el tratamiento T3 es a campo, sólo se encontró diferencia en la cantidad de $\alpha$-linolénico en la grasa intramuscular, no así para $\Sigma$ AGPI. 


\begin{tabular}{|l|c|c|c|c|}
\hline & T1 & T2 & T3 & P* $^{*}$ \\
\hline$\sum$ AGPI / AGGS & $0,30 \pm 0,03^{\text {a }}$ & $0,32 \pm 0,03^{\text {a }}$ & $0,30 \pm 0,03^{\text {a }}$ & 0,8128 \\
\hline n-6 / n-3 & $12,42 \pm 1,41^{\text {a }}$ & $12,61 \pm 1,41^{\text {a }}$ & $11,42 \pm 1,41^{\text {a }}$ & 0,1637 \\
\hline AI & $0,43 \pm 0,01^{\text {a }}$ & $0,43 \pm 0,01^{\text {a }}$ & $0,45 \pm 0,01^{\text {a }}$ & 0,4161 \\
\hline TI & $1,29 \pm 0,04^{\text {a }}$ & $1,27 \pm 0,04^{\text {a }}$ & $1,30 \pm 0,04^{\text {a }}$ & 0,8581 \\
\hline h/H & $2,33 \pm 0,05^{\text {a }}$ & $2,33 \pm 0,05^{\text {a }}$ & $2,26 \pm 0,05^{\text {a }}$ & 0,5593 \\
\hline
\end{tabular}

Tabla 6. Relaciones entre componentes de la grasa intramuscular utilizadas como indicadores de cualidades nutricionales $\left({ }^{\star} P\right.$ - Nivel de significancia del efecto del tratamiento en el modelo). Letras iguales dentro de una misma fila indican que no existe diferencia significativa entre las muestras para ese parámetro.

La determinación del AI y del TI propuestos por Ulbricht y Southgate (1991) no muestra diferencias estadísticamente significativas entre tratamientos. Estos índices ponderan los efectos de los diferentes ácidos grasos relacionados a la incidencia de enfermedades coronarias, tanto en lo que respecta a la promoción como en la protección de su incidencia.

La relación h/H es otro índice utilizado para estimar las cualidades nutricionales de los alimentos (Herranz et al., 2008). En este trabajo no se encontraron diferencias estadísticamente significativas entre los tratamientos para este indicador.

\begin{tabular}{|l|c|c|c|c|}
\hline & T1 & T2 & T3 & P* $^{*}$ \\
\hline Hierro (mg/100g) & $1,01 \pm 0,08^{\text {a }}$ & $0,96 \pm 0,08^{\text {a }}$ & $1,19 \pm 0,08^{\text {a }}$ & 0,1234 \\
\hline Zinc (mg/100g) & $2,24 \pm 0,16^{\text {a }}$ & $2,14 \pm 0,15^{\text {a }}$ & $2,37 \pm 0,16^{\text {a }}$ & 0,5179 \\
\hline Magnesio (mg/100g) & $21,50 \pm 0,52^{\text {a }}$ & $20,00 \pm 0,49^{\mathrm{b}}$ & $21,20 \pm 0,52^{\text {a, b }}$ & 0,1035 \\
\hline Sodio (mg/100g) & $44,90 \pm 1,97^{\text {a }}$ & $43,00 \pm 1,88^{\text {a }}$ & $45,60 \pm 1,97^{\text {a }}$ & 0,6153 \\
\hline Vit. E (mg $\alpha$-tocoferol/100g) & $1,25 \pm 0,15^{\text {a, b }}$ & $1,05 \pm 0,15^{\mathrm{b}}$ & $1,58 \pm 0,15^{\text {a }}$ & 0,0609 \\
\hline
\end{tabular}

Tabla 7. Contenido en minerales y vitamina E de la carne de cerdo en cada tratamiento estudiado ( ${ }^{*}$ - Nivel de significancia del efecto del tratamiento en el modelo). Letras iguales dentro de una misma fila indican que no existe diferencia significativa entre las muestras para ese parámetro.

No se hallaron diferencias estadísticamente significativas en el aporte de hierro, zinc y sodio entre los tratamientos estudiados y sus valores se encuentran dentro de lo esperado para los alimentos cárnicos según las Guías Alimentarias Basadas en Alimentos de Uruguay (MSP, 2005). La magnitud de las diferencias en el contenido de magnesio no es relevante desde el punto de vista nutricional, según la misma referencia. Los valores determinados para sodio, magnesio y zinc se encuentran dentro del rango verificado por Vautier (2006) para diferentes cortes comerciales de carne de cerdo; en el caso del hierro los valores se ubican por encima de los obtenidos por el autor.

En cuanto a vitamina E se observaron diferencias significativas entre los tratamientos T2 y T3, con el contenido mayor en el tratamiento T3. Este micronutriente es valorado por su comprobada capacidad antioxidante, como forma de aumentar la barrera antioxidante natural del consumidor (Elejalde, 2001).

Hoffman et al. (2003) no constataron efecto del sistema de producción (estabulado vs. a campo) en el contenido de minerales de la carne. Según Vautier (2005), no se ha podido establecer en forma consistente la existencia de un efecto de la cría a campo sobre el contenido de vitamina E.

\begin{tabular}{|c|c|c|c|c|}
\hline & T1 & $\mathbf{T 2}$ & T3 & $\mathbf{P}^{*}$ \\
\hline C16:0 & $22,90 \pm 0,50^{\mathrm{a}}$ & $23,90 \pm 0,50^{a}$ & $23,40 \pm 0,50^{\mathrm{a}}$ & 0,3257 \\
\hline C16:1 cis & $1,67 \pm 0,07^{\mathrm{a}}$ & $1,50 \pm 0,07^{\mathrm{b}}$ & $1,76 \pm 0,07^{\mathrm{a}}$ & 0,0511 \\
\hline C18:0 & $13,70 \pm 0,50^{\mathrm{a}}$ & $14,50 \pm 0,50^{\mathrm{a}}$ & $13,50 \pm 0,50^{a}$ & 0,2194 \\
\hline C18:1 cis & $43,80 \pm 0,70^{\mathrm{a}}$ & $43,10 \pm 0,70^{\mathrm{a}}$ & $42,60 \pm 0,70^{\mathrm{a}}$ & 0,5036 \\
\hline C18:2 n-6 & $12,30 \pm 0,60^{a}$ & $11,10 \pm 0,60^{a}$ & $12,50 \pm 0,60^{a}$ & 0,2546 \\
\hline C18:3 n-3 & $0,70 \pm 0,13^{a, b}$ & $0,39 \pm 0,13^{b}$ & $0,95 \pm 0,13^{\mathrm{a}}$ & 0,0171 \\
\hline$\sum$ Saturados (AGS) & $38,42 \pm 0,93^{\text {a }}$ & $40,94 \pm 0,98^{a}$ & $39,07 \pm 0,94^{\mathrm{a}}$ & 0,1734 \\
\hline$\sum$ Monoinsaturados (AGMI) & $47,36 \pm 0,68^{\mathrm{a}}$ & $46,56 \pm 0,71^{\mathrm{a}}$ & $46,27 \pm 0,68^{\mathrm{a}}$ & 0,5180 \\
\hline$\sum$ Poliinsaturados (AGPI) & $14,25 \pm 0,76^{\mathrm{a}}$ & $12,53 \pm 0,79^{a}$ & $14,65 \pm 0,76^{\mathrm{a}}$ & 0,1415 \\
\hline$\sum n-6$ & $12,51 \pm 0,62^{\mathrm{a}}$ & $11,33 \pm 0,65^{\mathrm{a}}$ & $12,69 \pm 0,62^{\mathrm{a}}$ & 0,2683 \\
\hline$\sum \mathbf{n - 3}$ & $1,38 \pm 0,17^{a, b}$ & $0,95 \pm 0,18^{b}$ & $1,59 \pm 0,17^{\mathrm{a}}$ & 0,0438 \\
\hline
\end{tabular}

Tabla 8. Perfil lipídico de la grasa subcutánea en cada tratamiento expresado en porcentaje (*P - Nivel de significancia del efecto del tratamiento en el modelo). Letras iguales dentro de una misma fila indican que no existe diferencia significativa entre las muestras para ese parámetro. 


\begin{tabular}{|c|c|c|c|c|}
\hline & $\mathbf{T 1}$ & $\mathbf{T 2}$ & T3 & $\mathbf{P} *$ \\
\hline AGPI / AGS & $0,38 \pm 0,03^{a}$ & $0,31 \pm 0,03^{a}$ & $0,38 \pm 0,03^{a}$ & 0,1115 \\
\hline$n-6 / n-3$ & $10,71 \pm 0,88^{a, b}$ & $12,32 \pm 0,92^{\mathrm{a}}$ & $8,83 \pm 0,88^{b}$ & 0,0326 \\
\hline AI & $0,45 \pm 0,02^{a}$ & $0,49 \pm 0,02^{\mathrm{a}}$ & $0,46 \pm 0,02^{a}$ & 0,3137 \\
\hline TI & $1,11 \pm 0,05^{\mathrm{a}}$ & $1,25 \pm 0,05^{\mathrm{a}}$ & $1,12 \pm 0,05^{\mathrm{a}}$ & 0,1290 \\
\hline $\mathbf{h} / \mathbf{H}$ & $2,59 \pm 0,09^{a}$ & $2,36 \pm 0,10^{a}$ & $2,51 \pm 0,09^{a}$ & 0,2033 \\
\hline Relación esteárico / linoleico & $1,14 \pm 0,09^{\mathrm{a}}$ & $1,32 \pm 0,09^{\mathrm{a}}$ & $1,13 \pm 0,09^{\mathrm{a}}$ & 0,2890 \\
\hline
\end{tabular}

Tabla 9. Relaciones entre componentes de la grasa subcutánea utilizadas como indicadores de cualidades nutricionales o aptitud tecnológica (*P - Nivel de significancia del efecto del tratamiento en el modelo). Letras iguales dentro de una misma fila indican que no existe diferencia significativa entre las muestras para ese parámetro.

El efecto de las pasturas en el incremento de contenido de ácido graso C18:3 n-3 $\alpha$ - linolénico en la grasa subcutánea, en el tratamiento T3, es consistente con resultados obtenidos por Basso et al. (2007) y Echenique et al. (2009) en cerdos con acceso a pasturas. No se encontraron diferencias significativas en el contenido de n-6 linoleico entre los tres tratamientos estudiados. Dado que otras fuentes alimenticias cubren las necesidades de este ácido graso, no es de interés nutricional aumentar su cantidad en alimentos cárnicos. En este sentido los tratamientos $\mathrm{T} 1$ y $\mathrm{T} 3$ fueron los que presentaron los mayores contenidos de C18:3 n-3 $\alpha$-linolénico, sin que se encontraran diferencias estadísticamente significativas entre ellos, pero sí entre los tratamientos $\mathrm{T} 2$ y T3. La ausencia de diferencias se podría explicar porque los tratamientos T1 y T3 comparten el $90 \%$ de la composición de la ración.

De igual modo, T2 y T3 presentaron diferencias estadísticamente significativas en la relación n-6/n-3 de la grasa subcutánea, lo que se explica por el aumento significativo de los ácidos grasos de la serie $\mathrm{n}$-3, especialmente el $\alpha$-linolénico. A pesar de que estos resultados son favorables desde el punto de vista nutricional, debe considerarse que el consumo de grasa subcutánea, salvo excepciones, se mantendrá como no recomendable por su elevado aporte de ácidos grasos saturados y concentración energética (OMS, 2008). Las relaciones AGPI/AGS y n-6/n-3 de la grasa subcutánea obtenidas en los diferentes tratamientos de este ensayo se ubican en un rango similar al determinado por Capra et al. (2007) para dietas formuladas con ingredientes con bajo aporte relativo de ácido linoleico. No se verificaron diferencias significativas en los otros índices utilizados para caracterizar las cualidades nutricionales de las grasas.

La comparación de la composición de las grasas subcutánea e intramuscular confirma que la composición de la grasa de la dieta tiene menor efecto sobre el perfil lipídico de la grasa intramuscular que sobre el tejido adiposo subcutáneo (Musella et al., 2009).

\section{Aptitud tecnológica de la grasa para la elaboración de salames}

En la elaboración de salames se analizó la actividad de agua de la pasta de salame, antes de su fermentación. Los resultados analíticos arrojaron altos valores previsibles, comprendidos entre 0,952 y 0,958 . Finalizado el producto y cuando se consideró apto para su comercialización, se realizaron los análisis de la actividad de agua, proteína y $\mathrm{pH}$ final. La a $\mathrm{a}_{\mathrm{w}}$ se situó entre 0,931 y 0,941 , el $\mathrm{pH}$ entre 4,66 y 4,73 y el nivel de proteínas entre 17,6 y $19,8 \%$ ( $\mathrm{f}=6,25)$.

Dalla Santa et al. (2006) establecen en su estudio que los salames poseen mayor o menor estabilidad en función de su actividad de agua y $\mathrm{pH}$. En dicho estudio se analizaron 50 muestras de salame y se presentan resultados con valores de $\mathrm{a}_{\mathrm{w}}$ que oscilan entre 0.80 y 0,95 , valores de $\mathrm{pH}$ entre 4,35 y 6,92 y valores de proteínas entre 11,32 y $41,27 \%$. De acuerdo al criterio establecido por estos autores, los salames obtenidos en este estudio pueden considerarse como un producto estable.

Debido a la importancia que reviste la aptitud tecnológica de la grasa para la producción de productos cárnicos, especialmente productos fermentados crudos sometidos a temperaturas moderadas, se debe considerar la relación existente entre ácidos grasos saturados e insaturados. Warnants et al. (1998) afirman que el nivel máximo recomendable de AGPI en la grasa utilizada para elaboración de salames se ubicaría en el entorno del $20 \%$, y que si el ácido linoleico es el AGPI predominante en la dieta del cerdo no cabe esperar efectos sensoriales indeseables. Sin embargo, Boulard et al. (1995) y LópezBote et al. (1999) señalan que en grasas destinadas a la elaboración de embutidos crudos madurados, el contenido límite de C18:2 sería del $12 \%$, que en condiciones excepcionales se podría aumentar hasta el $14 \%$. Vautier (2005) cita trabajos de Wood (1984) en los que se recomienda no sobrepasar el $15 \%$ de ácido linoleico en la grasa subcutánea, y de Mourot (2001), que sugiere un máximo de $12 \%$ de ácido linoleico y un mínimo de $12 \%$ de ácido esteárico. Echenique (2007) sostiene que los ácidos grasos que presentan una alta correlación con la consistencia de la grasa son los ácidos esteárico (C18:0) y linoleico (C18:2), y Whittington et al. (1986) hallaron que esta relación es la mejor forma de predecir la firmeza de la grasa y, por lo tanto, su comportamiento tecnológico. Se considera que una grasa es blanda cuando esta relación es inferior a 1,47 (Daza y Buxadé, 2000). En este trabajo esta relación se sitúa por debajo de ese índice y se ubica entre 1,05 y 1,23 para T1, 1,23 y 1,41 para T2 y entre 1,04 y 1,22 para T3. El contenido de ácido linoleico en los tres tratamientos se ubicó en valores próximos a $12 \%$, como se muestra en la Tabla 8 , lo que podría explicar el buen comportamiento térmico de la grasa a pesar de la baja relación entre esteárico y linoleico.

No se verificaron diferencias estadísticamente significativas en los valores de punto de fusión. Los valores obtenidos en este estudio son similares a los verificados por Galietta (2005) para cerdos producidos en confinamiento o en pastoreo, con un nivel leve de restricción de concentrado. Este parámetro es presentado a los industriales nacionales como indicador de la calidad de la grasa importada. Si bien Toldrá (2002) sugiere utilizar grasa con punto de fusión elevado en la elaboración de productos cárnicos fermentados, para evitar efectos adversos en el flavor (rancidez), este parámetro resulta insuficiente para predecir posibles defectos derivados de la fusión parcial de la grasa o una consistencia excesivamente oleosa en el feteado.

Los resultados del análisis sensorial de los salames se presentan en la Tabla 10.

\begin{tabular}{|c|c|c|c|}
\hline Tratamiento & Textura & Agrado general & Intención de compra \\
\hline T1 & $6,9 \mathrm{~b}$ & $6,5 \mathrm{a}$ & $5,2 \mathrm{~b}$ \\
\hline T2 & $6,2 \mathrm{a}, \mathrm{b}$ & $6,1 \mathrm{a}$ & $4,8 \mathrm{a}, \mathrm{b}$ \\
\hline $\mathbf{T 3}$ & $5,9 \mathrm{a}$ & $5,6 \mathrm{a}$ & $4,3 \mathrm{a}$ \\
\hline
\end{tabular}

Tabla 10. Análisis sensorial de los salames elaborados con la grasa de cada tratamiento. Letras iguales dentro de una misma columna indican que no existe diferencia significativa entre las muestras para ese parámetro. 
El 55,3\% de los consumidores prefirió la muestra elaborada con grasa del tratamiento T1, mientras que un 18,4\% prefirió en igual medida las muestras procedentes de los otros dos tratamientos. La preferencia de los consumidores por el tratamiento $\mathrm{T} 1$ con respecto al T3 se podría atribuir a la composición de la grasa, fundamentalmente a la diferencia en contenido en ácido linolénico, aunque las mismas son cuantitativamente muy estrechas. Se ha establecido que el incremento en el contenido de ácidos grasos poliinsaturados del grupo n-3 puede asociarse a sabores o aromas indeseables en las carnes (Wood et al., 2004). Bryhni et al. (2002) afirman que aunque altos niveles de AGPI pueden contribuir a una carne más saludable, sus efectos indeseables sobre las propiedades sensoriales pueden transformarse en limitante. Hoz et al. (2004) asocian estos efectos indeseables en productos fermentados crudos a la estabilidad oxidativa de los AGPI. Sin embargo, estos efectos se manifiestan con contenidos de C18:3 n-3 mayores a los obtenidos en este estudio.

\section{Conclusiones}

Los cerdos alimentados con ración de proteínas de origen vegetal y animal restringida al $90 \%$ y pasturas a voluntad (T3) presentaron un mayor contenido de ácido linolénico n-3 y de vitamina $\mathrm{E}$, comparados con los animales sometidos a los otros tratamientos. A pesar de la pequeña magnitud de la diferencia, desde el punto de vista nutricional esto representa una oportunidad dentro del grupo de alimentos cárnicos recomendables, ya que ofrece un doble beneficio al consumidor: mayor contenido de ácido linolénico, precursor de eicosanoides y del mantenimiento de la fluidez y permeabilidad de las membranas celulares, y mayor contenido de vitamina E, destacada por su efecto antioxidante. Los resultados alientan la posibilidad de mejorar el valor nutricional de la carne de cerdo por enriquecimiento en compuestos bioactivos a través del manejo de la dieta de los animales. Si bien la bibliografía internacional sobre esta temática es abundante, el desafío es lograr resultados nutricionalmente relevantes en base a los ingredientes disponibles en nuestras condiciones de producción. También es necesario considerar que en Uruguay no hay una producción de cerdos orientada hacia un destino específico (obtención de cortes frescos o elaboración de productos chacinados), sino que se busca lograr cualidades de la canal y de la carne que permitan satisfacer el doble propósito.

Desde el punto de vista tecnológico el comportamiento de la grasa utilizada fue acorde a lo esperado según los valores analíticos determinados. Los salames elaborados se consideran estables y con características técnicas y comerciales adecuadas, no presentando indicios de defectos por fusión de la grasa. Los resultados obtenidos sugieren que cuando se logra mantener los valores de ácido linoleico C18:2 en el entorno del $12 \%$, el comportamiento tecnológico de la grasa es adecuado, aunque la relación entre el contenido de los ácidos esteárico y linoleico sea inferior al valor recomendado.

Una adecuada formulación de las dietas para cerdos en crecimiento puede asegurar que la composición de las grasas destinadas a la elaboración de productos cárnicos fermentados se ajuste a las recomendaciones establecidas en la bibliografía. Este trabajo confirma que el objetivo puede ser logrado en las condiciones de producción prevalecientes en sistemas familiares típicos de Uruguay, con formulaciones basadas en suplementos proteicos de origen animal o exclusivamente vegetal, tanto en sistemas de engorde en estabulación o con acceso a pasturas.

La preferencia del panel de consumidores por el salame correspondiente al T1, tanto en textura como en intención de compra, sugiere la necesidad de profundizar el conocimiento sobre el efecto del contenido de AGPI y de C18:3 n-3 en la grasa utilizada sobre las propiedades sensoriales del producto final.

\section{Reconocimientos}

A los siguientes departamentos de LATU por su apoyo en análisis: Desarrollo de Métodos Analíticos, Cereales, Oleaginosos y Productos Derivados, Productos Lácteos, Cárnicos, Oleaginosos y de la Colmena.

A la empresa Antic S.A. por ceder sus instalaciones para el proceso de elaboración de los salames.

Al Dr. Gabriel Ciappesoni y la Ing. Agr. Andrea Ruggia, M.Sc. por su colaboración en los análisis estadísticos.

\section{Referencias}

- AMERICAN OIL CHEMIST'S SOCIETY. Official methods and recommended practices of the AOCS. Oficial Method Ce 2-66. Preparation of methyl esters of fatty acids. 6a ed. Urbana: AOCS, 2009.

- AMERICAN OIL CHEMIST'S SOCIETY. Official methods and recommended practices of the AOCS. Oficial Method Ce 1-62: Fatty acid composition by packed column gas chromatography. 6a ed. Urbana: AOCS, 2009.

- AMERICAN OIL CHEMIST'S SOCIETY. Official methods and recommended practices of the AOCS. Oficial Method Ce 1-91: Determination of fatty acids in edible oils and fats by capilary GLC. 4a. ed., reapproved 1993. Urbana: AOCS, 1995.

- AMERICAN OIL CHEMIST'S SOCIETY. Official methods and recommended practices of the AOCS. Oficial Method CC 1-25(93): Melting point capillary tube method Reapproved. 6a ed., reapproved 2009. Urbana: AOCS, 2009.

- AOAC INTERNATIONAL. Official Methods of Analysis of AOAC International. 17a ed., Gaithersburg: AOAC, 2000. Official Method 930.15.

- AOAC INTERNATIONAL. Official Methods of Analysis of AOAC International. 17a ed., Gaithersburg: AOAC, 2000. Official Method 988.05.

- AOAC INTERNATIONAL. Official Methods of Analysis of AOAC International. 17a ed., Gaithersburg: AOAC, 2000. Official Method 962.09.

- AOAC INTERNATIONAL. Official Methods of Analysis of AOAC International. 17a ed., Gaithersburg: AOAC, 2000. Official Method 920.39.

- AOAC INTERNATIONAL. Official Methods of Analysis of AOAC International. 17a ed., Gaithersburg: AOAC, 2000. Official Method 942.05.

- AOAC INTERNATIONAL. Official Methods of Analysis of AOAC International. 17a ed., Gaithersburg: AOAC, 2000. Official Method 927.02.

- AOAC INTERNATIONAL. Official Methods of Analysis of AOAC International. 17a ed., Gaithersburg: AOAC, 2000. Official Method 969.31.

- AOAC INTERNATIONAL. Official Methods of Analysis of AOAC International. 18a ed., Gaithersburg: AOAC, 2005. Official Method 999.10.

- BASSO, L.R.; MOISÁ, S.; BRUNORI, J.; FRANCO, R.; BACCI, R.; PAPOTTO, D. Calidad de carne diferencial de cerdos producidos en sistemas al aire libre. En: Agrociencia (vol. Especial). IX Encuentro de Nutrición y Producción de Monogástricos. 2007:63-68.

- BAUZÁ, R.; GIL, M.J.; PETROCELLI, H. Evaluación del comportamiento productivo de cuatro tipos genéticos de cerdos sometidos a los tres sistemas de alimentación más comúnmente utilizados en el país. En: INIA. Evaluación bioeconómica de sistemas de producción de cerdos. Montevideo: INIA, 2003. (Serie FPTA, 15). pp.110-146.

- BOULARD, J.; BOUYSSIERE, M.; CHEVILLON, P.; KERISIT, P.; LE-JOSSEC, P. Le tri des jambons selon la qualité des gras en salle de decoupe. En: Techni-Porc. 1995, 18: 21-32. 
- BRITISH STANDARD INSTITUTION (England). BS EN 12822: Foodstuffs. Determination of vitamin E by high performance liquid chromatography. Measurement of $\alpha-, \beta$-, $\gamma$ - and $\delta$ tocopherols. London: BSI, 2000.

- BRYHNI, E.A.; KJOS, N.P.; OFSTAD, R.; HUNT, M. Polyunsaturated fat and fish oil in diets for growing-finishing pigs: effects on fatty acid composition and meat, fat, and sausage quality. En: Meat Science. 2002, 62(1):1-8.

- CAPRA, G.; ECHENIQUE, A.; GROMPONE, M.A.; BAUZÁ, R.; GONZÁLEZ, A.; SILVA, D. Evaluación de la inclusión de grano de soja desactivada, afrechillo de arroz integral o suero de queso en la dieta de cerdos en engorde. 3 . Efecto sobre el perfil lipídico de la grasa subcutánea. En: Agrociencia (vol. especial). IX Encuentro de Nutrición y Producción en Animales Monogástricos. 2007:59-63.

- CORONADO, M.; VEGA Y LEÓN, S.; GUTIÉRREZ, R.; GARCÍA, B.; DÍAZ, G. Los ácidos grasos omega-3 y omega-6: nutrición, bioquímica y salud. En: Revista de Educación en Bioquímica. 2006, 25(003):72-79.

- DALLA SANTA, O.R.; COELHO, F.A.; FREITAS, J.R.S.; DALLA SANTA, H.S.; TERRA, N.S. Características de salames fermentados producidos sin adición de cultivo iniciador. En: Ciencia y Tecnología Alimentaria. 2006, 5(3):231-236.

- DAUMAS, G. Clasificación de las canales porcinas en Francia y Europa. En: $9^{\circ}$ Seminario Nacional de Desenvolvimiento da Suinocultura. Gramado: [s.n.], 2001. pp. 74-90.

- DAZA, A.; BOUXADÉ, C. La calidad de la carne del cerdo blanco. Producción porcina: aspectos claves. Madrid: MundiPrensa. 2000. pp. 237-257.

- DIEA-MGAP; INIA. Encuesta Porcina 2006. Caracterización de la situación productiva tecnológica, comercial y social del sector porcino. Montevideo: DIEA, 2007. (FPTA, 170).

- ECHENIQUE A. El efecto de la alimentación sobre la calidad de la carne y grasa de cerdo. En: IX Encuentro de Nutrición y Producción de Monogástricos. Cursos Pre Evento (Montevideo 14-16 de noviembre de 2007). Montevideo: [s.n.], 2007. pp. 55-63.

- ECHENIQUE, A.; CAPRA, G. Diagnóstico de la situación de la calidad de carne porcina para consumo fresco en Uruguay. En: INIA Serie Técnica. 2006, (160).

- ECHENIQUE, A.; CAPRA, G. Caracterización de los requerimientos de calidad de carne de cerdo por parte de las industrias cárnicas porcinas en Uruguay. En: INIA Serie Actividades de Difusión. 2007, (514).

- ECHENIQUE, A.; CAPRA, G.; PARDO, G.; GROMPONE, M.A.; URRUZOLA, N. Efecto de las pasturas sobre la composición química de la grasa intramuscular de cerdos machos enteros y castrados producidos al aire libre en Uruguay. En: EEZ. AIDA XIII Jornadas sobre Producción Animal (Zaragoza 12-13 de mayo de 2009). Tomo 2. Zaragoza: EEZ, 2009. pp. 622-624.

- ELEJALDE, J. Estrés oxidativo, enfermedades y tratamientos antioxidantes. En: Anales de Medicina Interna. 2001, 18(6):326335.

- ENFÄLT, A.CH.; LUNDSTRÖM, K.; HANSSON, N.L.; NYSTRÖM, P.E. Effect of outdoor rearing and sire breed (Duroc or Yorkshire) on carcass composition and sensory and technological meat quality. En: Meat Science. 1997, 45(1):1-15.

- ERREA, E. Evolución reciente y perspectiva de los suinos. En: MGAP-OPYPA. Anuario 2010. Montevideo: MGAP, 2010. pp. 89-98.

- FEDNA. Tablas FEDNA de ingredientes para piensos [En línea]. [s.l.]: [s.n.], 2003. [Consulta: 06 de mayo de 2011]. Disponible en: www.etsia.upm.es/fedna/tablas/htm

- FOLCH, J.; LEES, M.; SLOANE-STANLEY, G.H. A simple method for the isolation and purification of total lipids from animal tissues. En: Journal of Biological Chemistry, 1957, 226:497-509.

- GALIETTA, G. Calidad de la carne porcina. En: Jornada Taller Utilización de pasturas en la alimentación de cerdos. Facultad de Agronomía. Montevideo, diciembre 2005. p. 33-38.

- GROMPONE, M.A.; IRIGARAY, B. Composición y propiedades de la grasa de cerdo. En: Carnes y Alimentos. 2008, 9(26):11-19.
- HENRY, Y.; PEREZ, J.M.; SEVE, B. Alimentation des porcs en croissance. En: L'alimentation des animaux monogastriques: porc, lapin, volaille. Paris: INRA, 1984. pp. 49-66.

- HERRANZ, B.; ORDOÑEZ, J.A.; DE LA HOZ, L.; HIERRO, E.; SOTO, E., CAMBERO, M.I. Fatty acid composition of salami from different countries and their nutritional implications. En: International Journal of Food Science and Nutrition. 2008, 59(78): 607-618.

- HOFFMAN, L.C.; STYGER, E.; MULLER, M.; BRAND, T.S. The growth and carcass and meat characteristics of pigs raised in a free-range or conventional housing system. En: South African Journal of Animal Science, 2003, 33(3):166-175.

- HOZ, L.; D'ARRIGO, M.; CAMBERO, I.; ORDÓNEZ, J.A. Development of an n-3 fatty acid and $\alpha$-tocopherol enriched dry fermented sausage. En: Meat Science. 2004, 67(3): 485-495.

- IICA. Instituto Interamericano de Cooperación para la Agricultura. La agricultura familiar en los países del Cono Sur. [s.1.]: IICA, 2007.

- INAC. Instituto Nacional de Carnes. Dirección de Contralor de Mercado Interno. Volúmenes comercializados de carne destino abasto en Montevideo [En línea]. Montevideo: INAC, 2011. [Consulta: 05 de mayo de 2011]. Disponible en: http://www.inac. gub.uy/innovaportal/file/1882/1/volumenes\%20montevideo\%20 hasta\%20marzo\%202011.pdf

- INTERNATIONAL STANDARD ORGANIZATION (Switzerland). ISO 937: Meat and meat products. Determination of nitrogen content (Reference method). Geneva: ISO, 1978.

- INTERNATIONAL STANDARD ORGANIZATION (Switzerland). ISO 8589: Sensory analysis -- General guidance for the design of test rooms. Geneva: ISO, 1988.

- INTERNATIONAL STANDARD ORGANIZATION (Switzerland). ISO 11885: Water quality -- Determination of selected elements by inductively coupled plasma optical emission spectrometry (ICP-OES). Geneva: ISO, 1996.

- LÓPEZ-BOTE, C.; ISABEL, B.; REY, A.I. Efecto de la nutrición y del manejo sobre la calidad de la grasa en el cerdo. En: FEDNA. $X V$ Curso de Especialización. Avances en nutrición y alimentación animal. Madrid: FEDNA, 1999.

- MSP. PROGRAMA NACIONAL DE NUTRICIÓN. Guias alimentarias basadas en alimentos de Uruguay: manual para la promoción de prácticas saludables de alimentación en la población uruguaya. Montevideo: MSP, 2005.

- MOUROT, J.; HERMIER, D. Lipids in monogastric animal meat. En: Reprod. Nutr. Dev. 2001, 41:109-118. Citado en: VAUTIER, A. Les valeurs nutritionnelles de la viande de porc: les facteurs de variation [En línea]. París: Institut Technique du Porc, 2005. [Consulta: 05 de mayo de 2011]. Disponible en: www.officeelevage.fr/dei/f-707a.pdf

- MUSELLA, M.; CANNATA, S.; ROSSI, R.; MOUROT, J.; BALDINI, P.; CORINO, C. Omega-3 polyunsaturated fatty acid from extruded linseed influences the fatty acid composition and sensory characteristics of dry-cured ham from heavy pigs. En: Journal Animal Science. 2009, 87(11):3578-3588.

- NRC. Nutrient requirements of swine. [s.1.]: National Academies Press, 1998.

- OMS. Prevención y control de las enfermedades no transmisibles. aplicación de la estrategia mundial. OMS: Vienna, 2008. (122 ${ }^{\mathrm{a}}$ reunión Consejo Ejecutivo EB122/9. Punto 4.6 del orden del día provisional). pp. 1-24.

- SAS INSTITUTE. SAS. Version 9.1.3. Cary, SAS Institute, 2003

- TOLDRÁ, F. Dry-cured meat products. Ed. Food \& Nutrition Press, Connecticut. 2002. p. 65-66.

- ULBRICHT, T.L.V.; SOUTHGATE, D.A.T. Coronary heart disease: seven dietary factors. En: Lancet. 1991, 338:985-992.

- VAUTIER, A. Les valeurs nutritionnelles de la viande de porc: les facteurs de variation [En línea]. Paris: Institut Technique du Porc, 2005. [Consulta: 05 de mayo de 2011]. Disponible en: www.officeelevage.fr/dei/f-707a.pdf

- VAUTIER, A. Les valeurs nutritionnelles de la viande de porc: 
analyses sur 9 pieces UVC [En línea]. Paris: Institut Technique du Porc, 2006. [Consulta: 05 de mayo de 2011]. Disponible en: www. office-elevage.fr/vpc/11jsmtv/11JSMTV.pdf

- WARNANTS, N.; VAN OECKEL, M.J.; BOUCQUÉ, Ch. V. Effect of incorporation of dietary polyunsaturated fatty acids in pork backfat on the quality of salami. En: Meat Science. 1998, 49(4):435-445.

- WHITTINGTON, F.M.; PRESCOTT N.J.; WOOD J.D.; ENSER M. The effect of dietary linoleic acid on the firmness of backfat in pigs on $85 \mathrm{~kg}$ live weigth. En: Journal of the Science of Food and Agricultural. 1986, 37:753-761.

- WOOD, J.D. Fat deposition and the quality of fat tissue in meat animals. En: WISEMAN, J. Fats in Animal Nutrition. London: Butterworths, 1984. pp. 407-435. Citado en: VAUTIER, A. Les valeurs nutritionnelles de la viande de porc: les facteurs de variation [En línea]. París: Institut Technique du Porc, 2005. [Consulta: 05 de mayo de 2011]. Disponible en: www.officeelevage.fr/dei/f-707a.pdf

- WOOD, J.D.; RICHARDSON, R.I.; NUTE, G.R.; CAMPO, M.M.; KASAPIDOU, E.; SHEARD, P.R.; ENSER, M. Effects of fatty acids on meat quality: a review. En: Meat Science. 2004, 66(1): 21-32. 